\title{
HIMALAYA KALENDER 2018
}

\section{Kraft und Inspiration der Frauen des Himalaya}

Frauen im Himalaya und ihre spirituelle Kraft stehen 2018 im Mittelpunkt des Himalaya Kalenders von EcoHimal. Dabei geht es diesmal nicht um die internationalen Bergsteigerinnen - von Claude Kogan über Wanda Rutkiewicz bis zu Gerlinde Kaltenbrunner sondern um die starken Frauen, die in den Himalayaländern durch ihre Arbeit, Beharrlichkeit, Klugheit und auch Lebensfreude in vielen Fällen das soziale und wirtschaftliche Überleben der Familien ermöglichen.

Der Kalender erscheint mit zwölf Motiven im Format 30,5 mal 30,5 Zentimetern, wieder mit Zwischenblättern aus handgeschöpftem Lokta-Papier aus Nepal. Dabei handelt es sich um naturgeschöpftes Papier, das aus der trockenen Rinde der Loktapflanze gewonnen wird, die im nepalesischen Himalaya wächst. Nach 2000 Jahre alter Tradition wird diese Rinde nach der Ernte zweimal gekocht und mit einem Hammer geschlagen. Hat die Rinde lange genug gekocht und ist schließlich sehr weich, wird die Fasermasse in einen Holzrahmen gegossen und verteilt und anschließend in der Sonne getrocknet. Das Ergebnis ist ein besonders weiches, flexibles Gewebe, an dem man sich neben den schönen Photos jeden Monat aufs Neue im kommenden Jahr erfreuen kann.

Die Auflage des Himalaya Kalenders 2018 beträgt 700 Stück. Er kostet 30 Euro pro Stück zuzüglich Portokosten. Bei Abnahme ab zehn Stück besteht die Möglichkeit von Sonderkonditionen. Da die Produktionskosten zur Gänze von Sponsoren übernommen werden, gelangt der gesamte Verkaufserlös in die Projekte von EcoHimal. An oberster

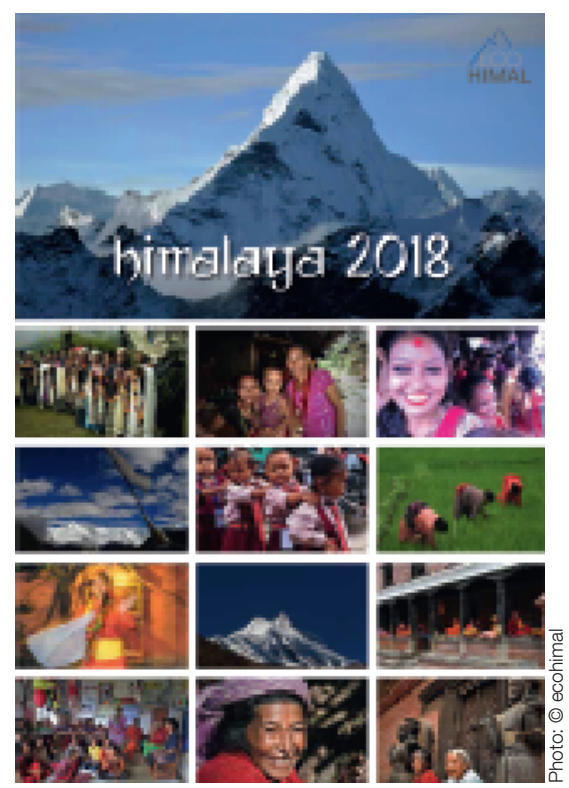
Stelle steht aktuell die Reparatur etlicher Wassersysteme, die in den Fluten des diesjährigen Monsun-Hochwassers zerstört wurden, und um in einigen Schulen Sanitäreinrichtungen zu errichten. Der Monsun ist im vergangenen Herbst mit großer Wucht über Südasien hereingebrochen und hat 100.000 e Quadratkilometer Ackerland meterhoch überflutet und die Ernten vernichtet. In Nepal sind mehr als 300 Menschen durch die Wassermassen ums Leben gekommen und 100.000 haben ihr Heim verloren.

Bestellungenan: office@ecohimal.org, k.luger@ecohimal.org, facebook/Ecohimal, Internet: www.ecohimal.org

\section{BUCH}

\section{Eine „Parallelaktion“ auf europäischer Ebene}

Die Geschichte vom Schwein zieht sich durch das gesamte Buch und zwar auf unterschiedliche und mehrheitlich sehr amüsante Weise. Im Mittelpunkt steht aber der europäische Gedanke, die Überwindung von nationalen Interessen und die vielen Gründe, Schachzüge und Absichten, die dieses Ziel in absehbarer Zeit nicht erreichbar scheinen lassen. Robert Menasse, präsente Stimme im heimischen Literaturbetrieb und moderater Mahner für das europäische Projekt, hat mit seinem jüngsten Buch „Die Hauptstadt“ einen geistreichen, spannenden und wunderbar verwobenen Roman über die Aktivitäten in der EU-Stadt Brüssel - und drumherum - geschrieben und wurde dafür ja auch kürzlich mit dem Deutschen Buchpreis ausgezeichnet.

Vier Jahre lang hat Menasse die Mechanismen des EU-Betriebs recherchiert, hat sich in Brüssel eine Wohnung gemietet, mit den EU-Beamten gesprochen, mit den Menschen gelebt. Damit hat er offenbar einen guten Einblick erhalten und „Die Hauptstadt" ist nun auch der erste Roman, der sich mit dieser Thematik befasst. Was den Autor selbst verblüfft hat, wie er in einem Interview bekannte. Denn die Schicksale, Verwicklungen und Vorgänge in diesem riesigen Betrieb der EUBürokratie haben allemal das Zeug für dramatische Zuspitzungen.

Zahlreiche Handlungsstränge entwickeln sich da. Eine karrierebewusste, griechischzypriotische Abteilungsleiterin in der EUKommission, die mit der ihr übertragenen Kultur-Agenda unzufrieden ist und eine Art „Parallelaktion"* auf die Beine stellen möchte, um Aufmerksamkeit und eine einflussreichere Abteilung zu gewinnen. Ein österreichischer Archäologe als Assistent ebendieser Abteilungsleiterin, dessen Bruder Schweinezüchter und Präsident der europäischen Interessensvertretung ist. Der wiederum kann nicht verstehen, warum die EU nicht in der Lage ist, mit den Chinesen einen Handelsvertrag über die sehr lukrative Ausfuhr von Schweinen nach China zu schließen. Denn die Schweineohren, die in unseren Breiten bestenfalls als Hundefutter verwendet werden, sind in China eine Delikatesse. Eine Liebesgeschichte in einem Seitenstrang der Geschichte zeigt eine emotionale Seite mit mehr oder minder romanti- scher Ausprägung. Eine logische Folge der oft intensiven Zusammenarbeit und manchmal vielleicht aus anderen Gründen. Auch einen Mord gibt es, an dessen Aufklärung offenbar die politischen

Stellen kein Interesse haben und die daher nicht nur den zuständigen Kommissar von dem Fall abziehen, sondern gleich alle Unterlagen und Informationen vernichten lassen. Die Stadtarchitektur und die lukullische Seite von Brüssel, philosophische, historische und gesellschaftspolitische Überlegungen der einzelnen Protagonisten - all das verpackt Menasse gekonnt und lustvoll in dieses 450 Seiten Werk und bereitet insgesamt ein großes und kluges Lesevergnügen.

Robert Menasse: Die Hauptstadt

Gebunden, 459 Seiten

ISBN: 978-3-518-42758-3

Suhrkamp Verlag, Berlin 2017

Preis: $24,70 €$

siehe dazu Robert Musils „Mann ohne Eigenschaften" 